\title{
Experimental test of the time stability of the half-life of alpha-decay ${ }^{214}$ Po nuclei
}

\author{
E.N. Alexeyev* Ju.M. Gavriljuk, A.M. Gangapshev, A.M. Gezhaev, \\ V.V. Kazalov, V.V. Kuzminov $\dagger$ and S.P. Yakimenko \\ Baksan Neutrino Observatory INR RAS, Russia \\ S.I. Panasenko and S.S. Ratkevich \\ V.N.Karazin Kharkiv National University, Ukraine
}

(Dated: May 26, 2011)

\begin{abstract}
A method and results of an experimental test of the time stability of the half-life of alpha-decay ${ }^{214}$ Po nuclei are presented. Two underground installations aimed at monitoring the time stability have been constructed. Time of measurement exceeds 1038 days for one set up and 562 days for the other. It was found that amplitude of possible annual variation of ${ }^{214}$ Po half-life does not exceed $0.2 \%$ of the mean value. The limit on the deviation of the decay curve from exponent at $0.034 \cdot T_{1 / 2}<t<0.1 \cdot T_{1 / 2}$ range has been found.

PACS numbers: 23.60.+e, 03.65.Xp
\end{abstract}

\section{INTRODUCTION}

A number of investigations aimed to check absolute stability of half-life of different radioactive isotopes and to search for possible time variations of the half-life constants under action of known and possible unknown natural factors has been carried out during last years.

In work [1] it was shown that, after many years of investigations with scintillation and semi-conductor $\alpha$ ray detectors, a conclusion was made about changes of rate of radioactive elements' $\beta$-decay with 24 hours and 27 days periodicities.

Many years measurement's decay rates data for the ${ }^{32} \mathrm{Si}(\beta$-decay) measured in the Brookhaven $\mathrm{Na}$ tional Laboratory (USA, 1982-1986 years) and for the ${ }^{226} \mathrm{Ra}(\alpha-$ and $\beta$-decays) measured in the PhysikalischTechnische-Bundesanstalt (Germany, 1983-1998 years) were analyzed in work [2], 3], [4]. Decay rate variations with a one year period and maximum amplitude of $\sim 0.15 \%$ in January-February were found in both data sets. The authors considered an assumed seasonal variations of the detector systems characteristics and/or the direct annular modulation of the count rate being caused by some unknown factor depending on the Sun-Earth distance as possible causes of such count rate variations.

The weak point of the count rate long time monitor experiments is a possible influence of meteorological, climatic and geophysical factors on the count rate of a source-detector pair. This shortcoming could be practically totally avoided in measurements based on a direct registration of a nuclear life time between birth and decay. Such a method allows us to answer the question of possible change with time of a just nuclear decay constant.

\footnotetext{
* alexeyev@ms1.inr.ac.ru

$\dagger$ bno_vvk@mail.ru

$\ddagger$ ratkevich@univer.kharkov.ua
}

Besides that a direct registration of nuclear life time allows us to study of the exponentially decay low. Some theoretical models [5], [6] predicted that decay curve does not exactly follow an exponential low in the short-and long-time regions in particular due to of the so-called quantum Zeno effect [7], 8], [9], [10]. Experimentally Zeno effect was proved [1] in repeatedly measured twolevel system undergoing Rabi transitions, but not observed in spontaneous decays.

Very important condition for measure possible deviations from an exponential low is the all investigated nuclei could have the same age.

\section{EXPERIMENTAL METHOD}

A primary task of the investigation presented in this work was to investigate constancy of a half-life $\tau$ ( $\tau \equiv$ $T_{1 / 2}$ ) of ${ }^{214}$ Po during several years. ${ }^{214}$ Po decays with $164.3 \mu$ s half-life [12] by emitting the $7.687 \mathrm{MeV} \alpha$ particle. This isotope appears mainly in the exited state $(\sim 87 \%)$ in the ${ }^{214} \mathrm{Bi} \beta$-decay. Half-lives of the exited levels does not exceed 0.2 ps [13] and they discharge instantly with regard to the scale of the ${ }^{214}$ Po half-life. Energies of the most intensive $\gamma$-lines are equal to 609.3 $\mathrm{keV}$ (46.1\% per decay), $1120 \mathrm{keV}(15.0 \%)$ and $1765 \mathrm{keV}$ $(15.9 \%)$. So, the $\beta$-particle and $\gamma$-quantum emitted at the moment of a birth of ${ }^{214}$ Po nuclear (start) and the $\alpha$-particle emitted at the decay moment (stop). Measurement of "start-stop" time intervals allows one to construct decay curve at an observation time and to determine the half-life from it's shape.

${ }^{214} \mathrm{Bi}$ isotope is an intermediate daughter product in the ${ }^{238} \mathrm{U}$ series. It gives almost all $\gamma$-activity of the series. This isotope could be produced with a constant velocity if an intermediate isotope ${ }^{226} \mathrm{Ra}\left(T_{1 / 2}=1600 \mathrm{y}\right)$ of the ${ }^{238} \mathrm{U}$ series will be used as a source. A time of an equilibrium reached in a partial ${ }^{226} \mathrm{Ra}^{214} \mathrm{Bi}$ series equals to $\sim 20$ days and is determined by the longest half-life isotope, ${ }^{222} \mathrm{Rn}$ (3.82 days). A ${ }^{214} \mathrm{Bi}$ decay rate doesn't change 


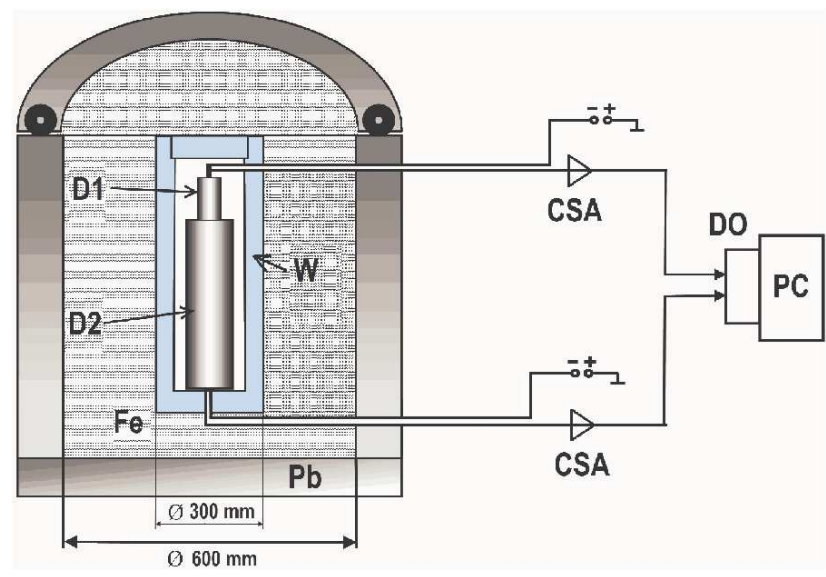

FIG. 1. Schematic cross-sectional view of TAU-1 setup and electronics block diagram.

after this time if a source is hermetically sealed to prevent an escape of the radon.

Two test sources with ${ }^{226} \mathrm{Ra}$ activity of $\sim 90 \mathrm{~Bq}$ and $\sim 20 \mathrm{~Bq}$ were prepared in the V.G. Khlopin Radium Institute (St.Petersburg, Russia) in March, 2008.

A thin transparent radium spot is deposited in the center of a polished plastic scintillator disc of $18 \mathrm{~mm}$ diameter and $0.8 \mathrm{~mm}$ thickness. A disc's side with the spot is covered by a similar disc hermetically glued on periphery $(2.5 \mathrm{~mm}$ width). A plastic scintillator (PS) registers all charged particles generated in the decay series. A PS light yield for an $\alpha$-particle absorption is $\sim 0.1$ of that for the electron of the same energy. Due to this fact $\alpha$ spectra mix with $\beta$-spectra if a scintillator is thick enough to absorb a complete energy of an electron. To prevent this effect and separate these spectra the scintillator discs were made thin enough so that electrons lose only part of their energy.

Two setups named TAU-1 and TAU-2 were made to measure radiations of these sources. A schematic sectional view of the TAU-1 with the electronics is shown in Fig1. The detector D1 contains the photomultiplier (FEU-85) which views the source disc through a plastic light guide. A teflon reflector is placed under the source to improve light collection. The whole assembly is packed in a stainless steel body. The detector D1 is placed on the butt-end of the scintillation detector D2 with low background $\mathrm{NaI}(\mathrm{Tl})$ crystal of $80 \mathrm{~mm}$ diameter and $160 \mathrm{~mm}$ length. The crystal has a stainless still cover and a quartz entrance window. The crystal with photomultiplier (FEU-110) is packed in a cooper body. The two detectors are mounted vertically in a low background shield made from $\mathrm{Pb}(10 \mathrm{~cm})+\mathrm{Fe}(15 \mathrm{~cm})+\mathrm{W}(3$ $\mathrm{cm})$. The TAU-1 is located in the underground laboratory "KAPRIZ" of the Baksan Neutrino Observatory of INR RAS at $1000 \mathrm{~m}$ of water equivalent depth. A cosmic ray background is decreased by $\sim 10^{4}$ times due to the rock thickness in comparison with the ground surface one. Walls of the laboratory are covered by a low background concrete thus decreasing by $\sim 8$ times the $\gamma$-ray background from a rock natural radioactivity. Total background suppression inside the shield is equal to $\sim 2000$ times.

Signals from the photomultipliers are read by charge sensitive preamplifiers (CSA) and fed to two inputs of a digitizer (digital oscilloscope - DO) LA-n20-12PCI which is inserted into a personal computer (PC). Pulses are digitized with $6.25 \mathrm{MHz}$ frequency. The DO pulse recording starts by a signal from the $\mathrm{D} 2$ which registered ${ }^{214} \mathrm{Bi}$ decay's $\gamma$-quanta. A D2 signal opens a record of a sequence with $655.36 \mu$ s total duration where first $81.92 \mu$ s time is a "prehistory" and the last $573.44 \mu \mathrm{s}$ is a "history". Duration of a "history" exceeds the three ${ }^{214}$ Po half-lives.

A PS disc source in the TAU-2 installation is fixed at the end of an air light guide having a smooth wall made of VM-2000 light reflecting film. The light guide is put into $0.8 \mathrm{~mm}$ thick stainless still rectangular frame with inner dimensions of $150 \cdot 23 \cdot 9 \mathrm{~mm}$. An open butt-end of the frame is welded into a bottom of cylindrical stainless still body of $45 \mathrm{~mm}$ diameter and $165 \mathrm{~mm}$ length where photomultiplier (FEU-85) placed. So much for the detector D1 of the TAU-2. Two scintillation detectors (D2a and D2b) made of large $\mathrm{NaI}(\mathrm{Tl})$ crystals $(150 \cdot 150 \mathrm{~mm})$ are used for a registration of the $\gamma$-quanta. Each crystal has a stainless still cover and a quartz entrance window. Photomultipliers (FEU-49) are used for the light registration. The D1 are installed into a gap between D2a and D2b. A scheme of pulse registration in the TAU2 is similar to the one of TAU-1 but signals from D1a and $\mathrm{D} 2 \mathrm{~b}$ are preliminarily summed in additional summer. The TAU-2 is located in the low background room in the deepest underground laboratory NLGZ-4900 of the BNO INR RAS at the $4900 \mathrm{~m}$ of water equivalent depth. A cosmic ray background is decreased by $\sim 10^{7}$ times by the rock thickness in comparison with the ground surface one. The room walls are made of polyethylene $(25 \mathrm{~cm})+$ $\mathrm{Cd}(0.1 \mathrm{~cm})+\mathrm{Pb}(15 \mathrm{~cm})$. The detectors are surrounded with additional lead shield of $15 \mathrm{~cm}$ thickness aimed to absorb a radiation from decays of ${ }^{214} \mathrm{Bi}$ in the air being in equilibrium with the radon in the room.

\section{WORKING CHARACTERISTICS}

Working characteristics of each installation were tested preliminary by using usual multichannel analyzer AI1024.

\section{$T A U-1$ installation}

An amplitude spectrum, from the D1, collected during $600 \mathrm{~s}$ is shown in Fig.2. Integral count rate is equal to $179 \mathrm{~s}^{-1}$. A wide peak above the 500 channel corresponds to ${ }^{214} \mathrm{Po} \alpha$-particles. A count rate within $500 \div 750$ channels is equal to $21.2 \mathrm{~s}^{-1}$. An amplitude spectrum, from the D2, collected during $30 \mathrm{~min}$ is shown in Fig 3 a. A main part of the spectrum is due to $\gamma$-quanta from the source. A spectrum of the D2 background was measured in 72 hours when the D1 was removed from the shield. 


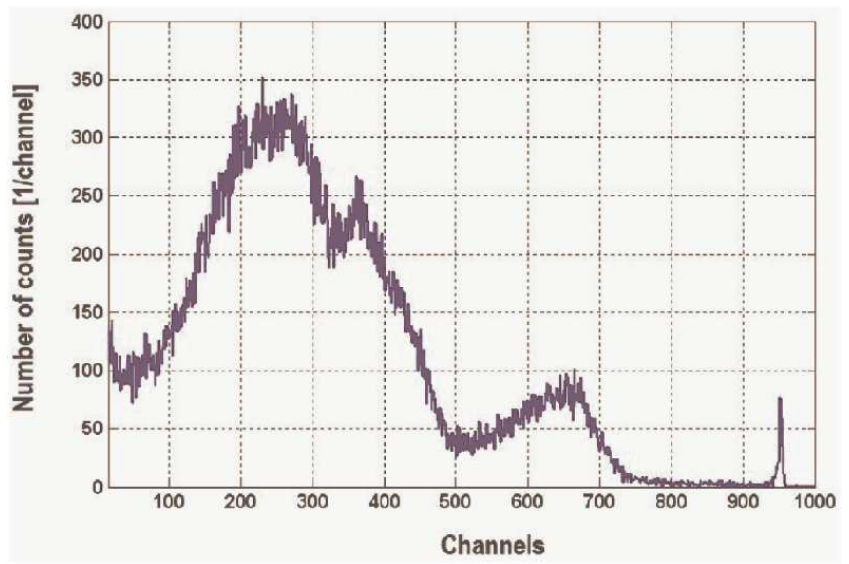

FIG. 2. D1 detector pulse amplitude spectrum collected at $600 \mathrm{~s}$.

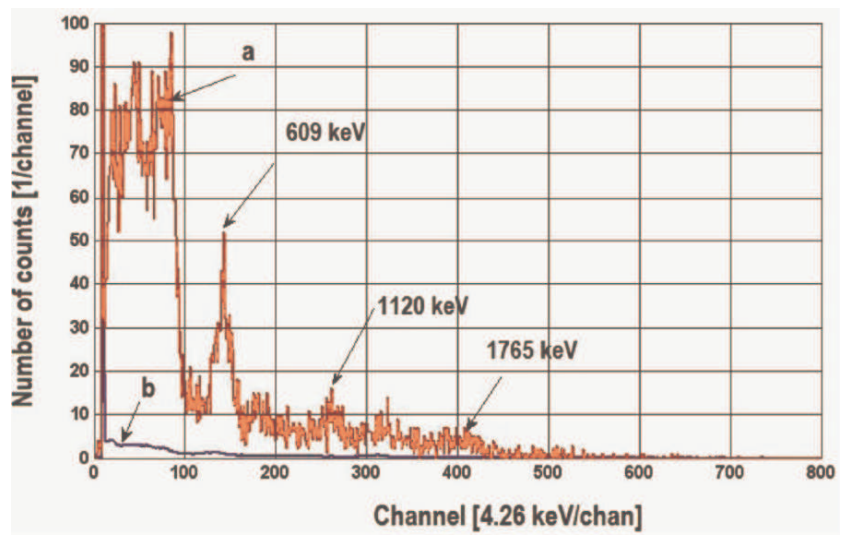

FIG. 3. D2 detector pulse amplitude spectra (a) of ${ }^{226} \mathrm{Ra}$ source (30 min collection time ) and (b) of background normalized at $30 \mathrm{~min}$ (72 hours collection time).

It's spectrum shown in the Fig.3b. An integral count rate in the spectrum in Fig $3 \mathrm{a}$ is $4.90 \mathrm{~s}^{-1}\left(1.64 \mathrm{~s}^{-1}\right.$ at the energy above $400 \mathrm{keV}$ ) and that one in Fig $3 \mathrm{~b}$ is 0.20 $\mathrm{s}^{-1}$.

\section{TAU-2 installation}

Integral count rate of the D1 is equal to $70 \mathrm{~s}^{-1}$. A background count rate of the D2a detector at the energy above $400 \mathrm{keV}$ is equal to $2.3 \mathrm{~s}^{-1}$. A total count rate with the source is equal to $5.0 \mathrm{~s}^{-1}$. A light collection coefficient of the D1 in the TAU-2 was found to be 0.51 of the one in the TAU-1.

\section{USEFUL EVENT SELECTION}

The TAU-1 and TAU-2 installations were placed into underground low background shields to provide stable environmental conditions and a low cosmic ray background. It allows us to decrease possible variations of electronics characteristics and to exclude random D1-D2 coincidences caused by D2 registrations of background's muons

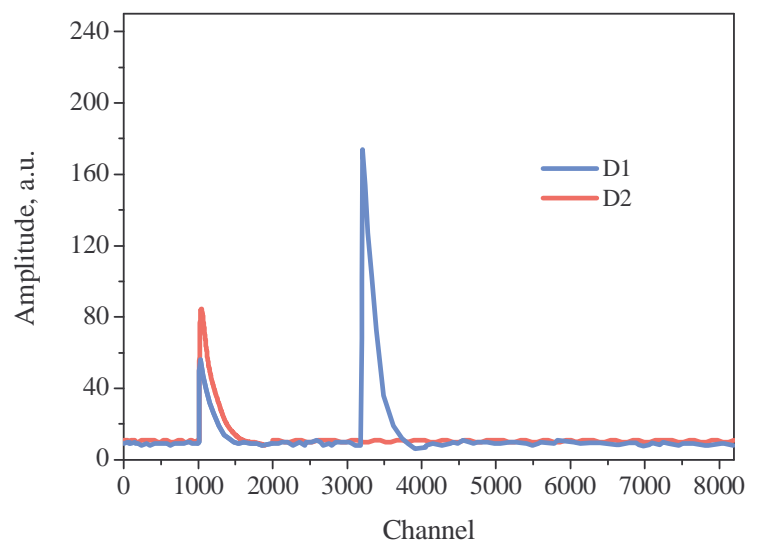

FIG. 4. An example of coinciding D1-D2 events with two pulses in the D1 "history" (blue) and one pulse in the D2 "history" (red).

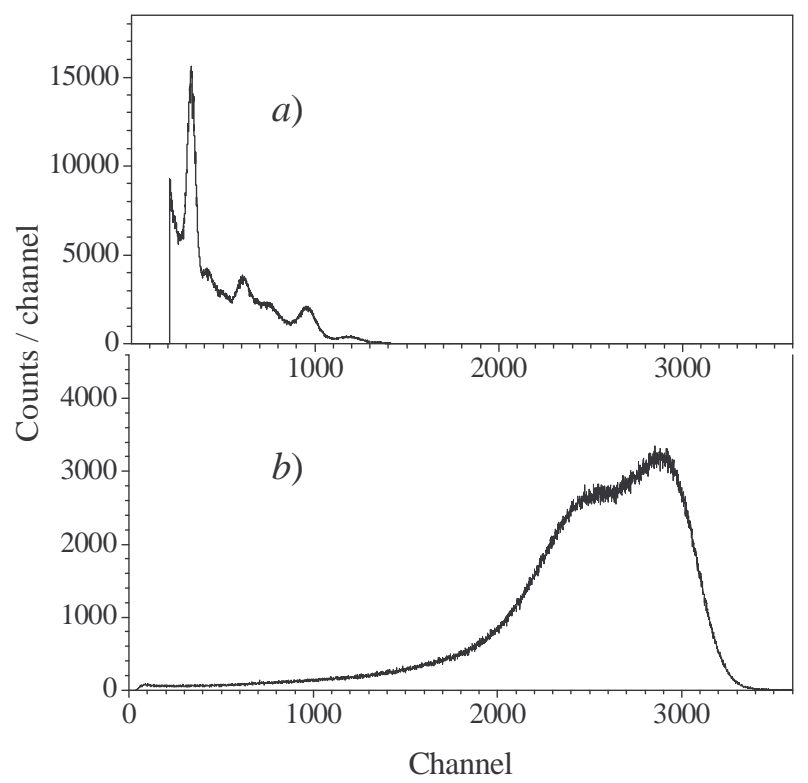

FIG. 5. Spectra of the D2 $\gamma$-quantum pulses (a) and the second D1 $\alpha$-particle pulses (b) of the TAU-1 setup.

and $\gamma$-quanta.

A control of the DO work in regimes of on-line event separation, data collection and visualization was carried out by specially designed PC program. This program allows DO to record only coinciding D1-D2 events, with two pulses in the D1 "history" and one pulse in the D2 "history". The last one is in prompt coincidence with the first D1 pulse. An example of the event is shown in Fig.4. A number of such events make up 48\% of the total amount. Their count rates are equal to $\sim 4.3 \mathrm{~s}^{-1}$ for TAU-1 and $\sim 4.6 \mathrm{~s}^{-1}$ for TAU-2 for the energy threshold of $400 \mathrm{keV}$ in the channels of D2 detector. Spectra of the D2 pulses ( $\gamma$-quanta) and the second D1 pulses ( $\alpha$ particles) for the TAU-1 are shown in Fig.5(a,b). Double peak of the Fig 5 b spectrum can be explained by differ- 


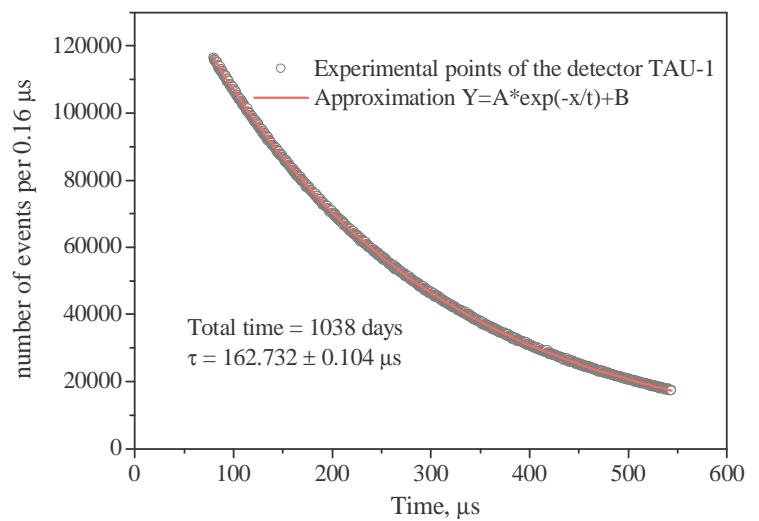

FIG. 6. Summary exponent constructed for the whole data set collected at 1038 days by TAU-1.

ent light output of the two PS disks. The lower energy peak was found to move towards the beginning of the coordinates with time. This effect could be connected with a degradation of PS surface scintillations properties under the active spot due to diffusion of chemical components or decay products into a surface layer of the source deposited disc.

\section{ON-LINE DATA PROCESSING}

A rate of the direct data record is $5.8 \mathrm{~Gb} \cdot$ day $^{-1}$. Such large information flux complicates processes of the data collection and off-line processing. A number of pulses and their delays for each event are analysed on-line to decrease usage of PC memory. "Wrong" events are excluded. Only a time of appearing and amplitude of pulses defined for the "right" events record at the PC memory. A rate of an information accumulation decreased up to $8.5 \mathrm{Mb} \cdot \mathrm{day}^{-1}$. Measurements with the TAU-1 setup started in April, 2008 and the ones with TAU-2 started in July, 2009.

\section{RESULTS AND DISCUSSION}

A continuous data set is divided on a sequence of equal intervals. A spectrum of delays between the first and second D1 pulses is constructed for each interval. This spectrum is approximated by exponential function viewed as $y=a \cdot \exp (-\ln (2) \cdot t / \tau)+b$ corresponding to the decay curve. A value of the half-life $\tau$ is found from this curve. The series of $\tau$-values is analysed to find out any regularity or variation.

Duration of the analyzed data sets of TAU-1 and TAU2 were equal to 1038 days and 562 days correspondingly. A half-life value obtained for a decay curve constructed with the whole set of TAU-1 data was found to be $(162.733 \pm 0.104) \mu \mathrm{s}$. This curve is shown in Fig 6 , A half-life value obtained for the TAU-2 data was found to

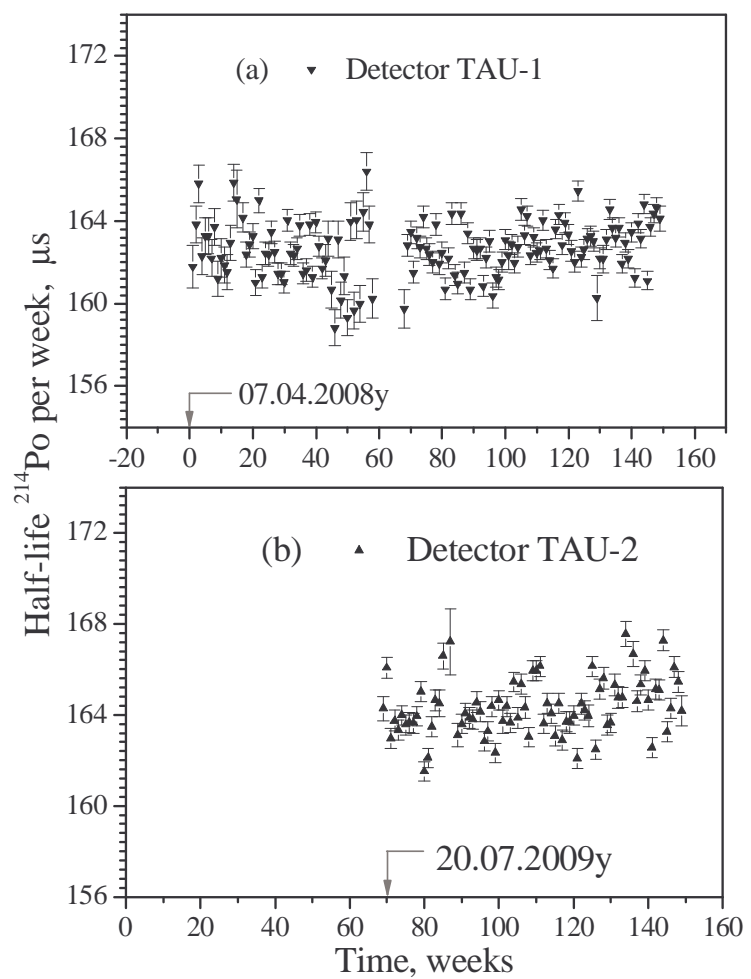

FIG. 7. Time dependence of ${ }^{214}$ Po half-life determined for one week collection interval data set from TAU-1 (a) and TAU-2 (b).

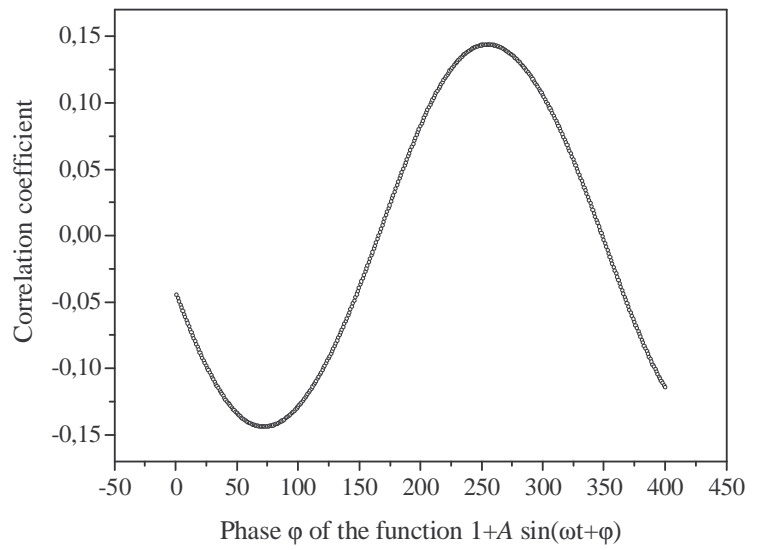

FIG. 8. Dependence of $k(\varphi)$ for the TAU-1 data.

be $(164.249 \pm 0.115) \mu \mathrm{s}$. The difference between values is connected with uncertainties of the digitizer's frequency calibrations.

Each interval of the whole data set was chosen to be 7 days for the analysis of the half-life constant behavior for a long time period. Sequences of $\tau$-values for TAU-1 and TAU-2 are shown in Fig 7 (a) and (b) respectively. To search for a possible annular variations of the data they were normalized on the averaged values and compared with a periodical function $f(t)=[1+A \cdot \sin (\omega t+\varphi)]$ where $\omega=1 / 365$ day $^{-1}, A$ and $\varphi$ are the amplitude 


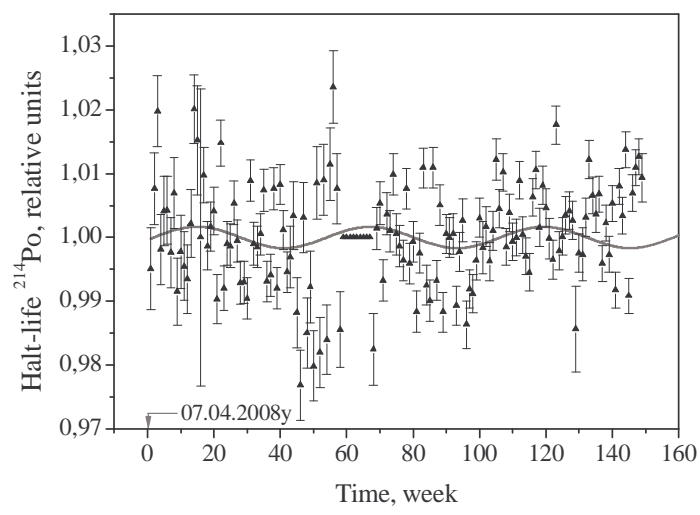

FIG. 9. Normalized graph of the time dependence of ${ }^{214} \mathrm{Po}$ half-life for TAU-1 in comparison with function $f(t)=[1+$ $0.00166 \cdot \sin (t / 365+255)], t$ - days.

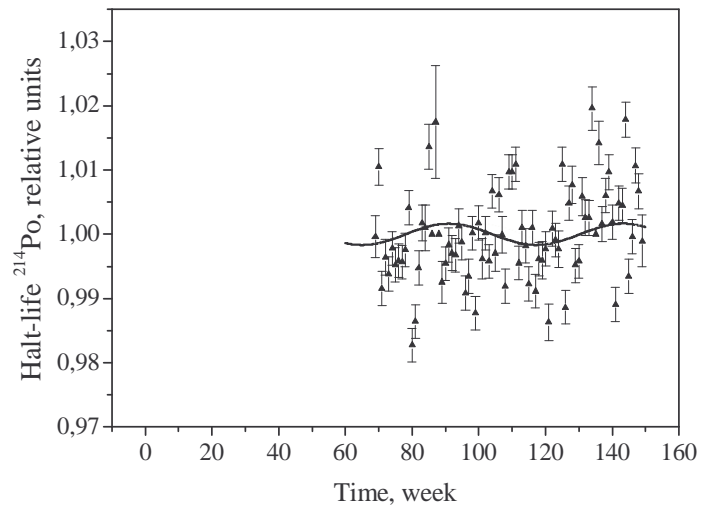

FIG. 10. Normalized graph of the time dependence of ${ }^{214} \mathrm{Po}$ half-life for TAU-2 in comparison with function $f(t)=[1+$ $0.00166 \cdot \sin (t / 365+88)], t$ - days.

and phase correspondingly. $A=0.002$ was taken at the beginning. A $\varphi$-value has been changed from 1 to 365 with the step of 1 day. A correlation coefficient $k$ between the $\tau$-sequences and $f(t)$ was calculated for the each $\varphi$ value. A dependence of $k(\varphi)$ for the TAU-1 data is shown in Fig, 8 .

The maximum value $k=0.144$ has been reached at $\varphi=225$. A selection of $A$-value corresponding to the $\chi^{2}$ minimum was done for this $\varphi$-value, and was found to be $A=0.00166$. The values of $k=0.160, A=0.00166$ and $\varphi=88$ days were found for TAU-2 by the similar way.

Corresponding graphs for the TAU-1 and TAU-2 data are shown in Fig. 9 and Fig[10. The estimated values of $A$ are the same for both setups but the phases differ by 167 days. A mean square error $s(k)$ of a $k$ for a number $N$ of degrees of freedom could be estimated as $s(k) \approx\left(1-k^{2}\right) / \sqrt{N}$. It was found that $s(k)=0.080$ for the TAU- $1(k=0.144$ and $N=149)$ and $s(k)=0.109$ for the TAU-2 $(k=0.160$ and $N=81)$.

A low reliability of the obtained results and disagreement between $\varphi$-values allow one to determine only the upper limit of the amplitude of $\tau$-constant possible annular variation which was found to be $A \leq 0.002(90 \%$

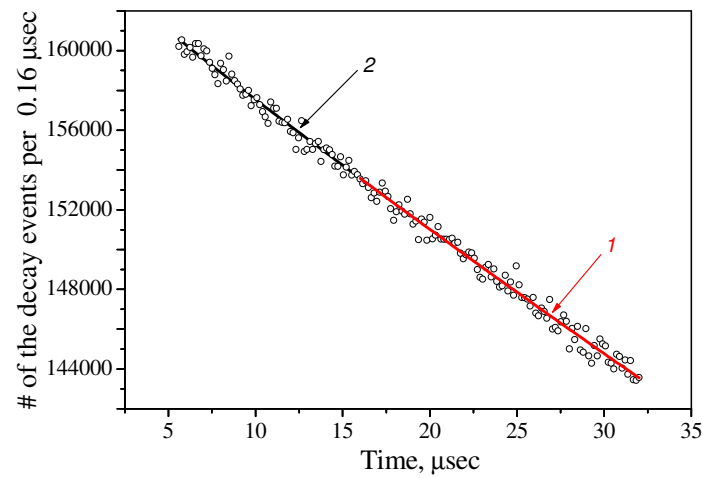

FIG. 11. The decay dependence of ${ }^{214} \mathrm{Po}$ of the detector TAU2. Points is the experimental data. Curve 1 is the result of the approximation of the exponential low. Curve 2 is the result of the aproximation using eq.1

C.L.). An interval of data spacing was chosen to be 1 day in order to search for possible short period variations. No significant frequency peaks in a range $0.003 \div 1.000$ day $^{-1}$ of the Fourier spectra constructed by using two such $T_{1 / 2}$ - series has been found.

We have tested the theoretical consideration of possible violation of the radioactive decay exponential law at low values of time of life $(t \lesssim 0.1 \tau)$ of ${ }^{214} \mathrm{Po}$ nucleus. For this purpose the total data set of the detector TAU-2 was used. The exponent was constructed for the time interval $5.6 \div 544 \mu$ sec. Half-live of the ${ }^{214}$ Po nucleus was determined using data for the time interval $16 \div 544 \mu$ sec. Then the residual part of the data was used for analysis of the deviations between the data and the exponent which was calculated.

The deviation was search for in form [5]:

$$
\exp (-t / \tau)+\sum_{i=1}^{n} a_{i}(t / \tau)^{-n}
$$

Limiting $\mathrm{n}=2$ we find: $\mathrm{a}_{1} \leqslant 4.01 \cdot 10^{-6}, \mathrm{a}_{2} \leqslant 3 \cdot 10^{-7}$, for time interval $\left(0.034 \cdot T_{1 / 2} \leqslant t \leqslant 0.1 \cdot T_{1 / 2}\right)$.

The results of the analysis are shown at the fig.11. The points at the figure are the experimental data for the time interval $5.6 \div 32 \mu \mathrm{sec}$. Curve 1 is the part of the exponent calculated for time interval $16 \div 544 \mu \mathrm{sec}$. Curve 2 is the result of the approximation of the data for time interval $5.6 \div 16 \mu$ sec, using eq. (1).

\section{CONCLUSIONS}

1. Two underground installations aimed at monitoring the time stability of ${ }^{214}$ Po half-life have been constructed.

2. Time of measurement exceeds, at present, 1038 days for TAU- 1 and 562 days for TAU-2.

3. It has been found that amplitude of possible annual variation of ${ }^{214} \mathrm{Po}$ half-life does not exceed $0.2 \%$ of the mean value. 
4. No deviation of the decay curve from exponent at $0.034 \cdot T_{1 / 2}<t<0.1 \cdot T_{1 / 2}$ has been found with an accuracy of $\sim \cdot 10^{-6}$.
[1] Yu.A. Baurov, Yu.G. Sobolev, Yu.V. Ryabov, V.F. Kushniruk, "Experimental Investigations of Changes in the Rate of Beta Decay of Radioactive Elements". Phys.of Atomic Nucl. V.70, No.11, 1825 (2007).

[2] Jere H. Jenkins at al., "Evidence for Correlations Between Nuclear Decay Rates and Earth-Sun Distance". Astropart. Phys. 32, 42 (2010).

[3] Peter A. Sturrock at al., "Concerning the Phases of Annual Variations of Nuclear Decay Rates". Astrophysical Journal 737, 65 (2011).

[4] Jere H. Jenkins at al., "Analysis of Experiments Exhibiting Time-Varying Nuclear Decay Rates: Systematic Effects or New Physics?". arXiv:1106.1678 [nucl-ex]; "Evidence for Time-Varying Nuclear Decay Rates: Experimental Results and Their Implications for New Physics" arXiv:1106.1470 [nucl-ex].

[5] P.M.Gopych and I.I.Zaljubovsky, "Exponentiality of the basic law of radioactive decay". PEPAN, 19, 4, 785, (1988).

[6] L. Fonda, G.C. Ghirardi and A. Rimini, "Decay theory of unstable quantum systems". Reports on Progress in Phisics 41, 587 (1978).

[7] L.A. Khalfin, "Zeno's quantum effect". Physics-Uspekhi 33, 10, 868 (1990).

[8] B. Misra and E.C.G. Sudarshan, "The Zeno's paradox in quantum theory". J.Math.Phys. 18, 756 (1977).

[9] P. Facchi and S. Pascasio, "Quantum Zeno dynamics: mathematical and physical aspects". J.Phys. A: Math.Teor. 41 (2008) 493001.

[10] Francesco Giacosa and Giuseppe Pagliara,"Oscillations in the decay law: a possible quantum mechanical explanation of the GSI anomaly". arXiv:1110.1669 [nucl-th].

[11] Wayne M. Itano, D. J. Heinzen, J. J. Bollinger, and D. J. Wineland, "Quantum Zeno effect". Phys.Rev. A41, 2295 (1990).

[12] E. Browne, Nuclear Data Sheets 99, 649 (2003).

[13] Table of Isotopes, Edited by R.B. Firestone et al., 8th ed., (Willey, New York, 1996).

[14] Physical Quantaty Data-book. (in Russian). Under I.K.Kikoin edition. M. Atomizdat. 1976. 


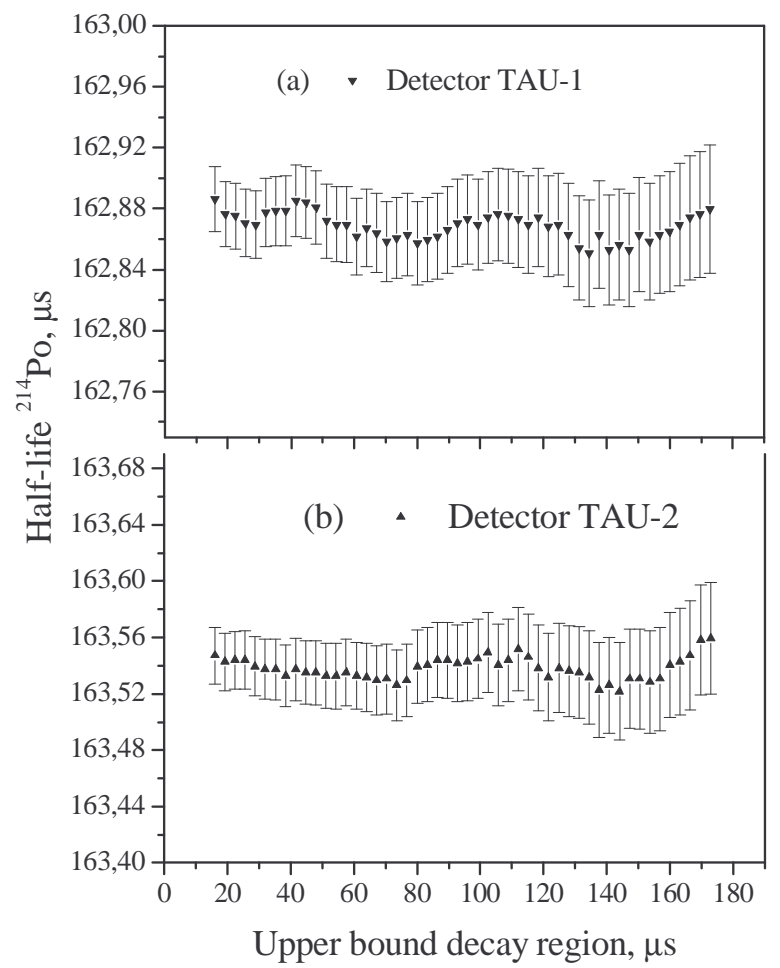

